\title{
Therapie der chronischen Entzündungsanämie - Eisen, Bluttransfusionen oder Erythropoetin?
}

\section{Treatment of the Anaemia of Chronic Disease - Iron, Transfusions or Human Recombinant Erythropoietin?}

\author{
Autor \\ J. P. Kaltwasser \\ Institut \\ Gemeinschaftspraxis für Orthopädie/Rheumatologie, Frankfurt am Main
}

\author{
Schlüsselwörter \\ - Entzündungsanämie (ACD) \\ - Erythropoetin \\ - funktioneller Eisenmangel \\ - rheumatoide Arthritis \\ Key words \\ - anaemia of chronic disease \\ (ACD) \\ - erythropoietin \\ - functional iron deficiency \\ - rheumatoid arthritis
}

\begin{abstract}
Bibliografie
DOI 10.1055/s-0029-1220676 Akt Rheumatol 2009; 34: 109-115 @ Georg Thieme Verlag KG Stuttgart · New York ISSN 0341-051X
\end{abstract}

\footnotetext{
Korrespondenzadresse Prof. Dr. Joachim Peter Kaltwasser

Gemeinschaftspraxis für Orthopädie/Rheumatologie Oeder Weg 2-4 60313 Frankfurt am Main Tel.: +69/36/60 1677 Fax: +69/36/601671 Prof.Dr.P.Kaltwasser@ t-online.de
}

\section{Zusammenfassung \\ $\nabla$}

Die Entzündungsanämie (ACD) ist ein Begleitphänomen vieler chronischer infektiöser, neoplastischer und autoimmunologischer Erkrankungen. Wo immer möglich steht die Sanierung der zugrunde liegenden Erkrankung als erste Option der Therapie im Vordergrund. Wo dies nicht möglich ist, stehen heute als therapeutische Möglichkeiten Bluttransfusionen, Eisenersatztherapie und die Stimulierung der Erythropoese mit Erythropoetinen zur Verfügung. Bluttransfusionen sind als kurzfristige, in Bezug auf den Effekt zeitlich begrenzte Maßnahme den lebensbedrohlichen Anämiegraden $(\mathrm{Hb}<6,5 \mathrm{~g} / \mathrm{dl})$ und der perioperativen Versorgung vorbehalten. Eisensubstitution ist indiziert wenn die ACD von einem Eisenmangel mit komplett entleerten Eisenspeichern begleitet wird. Eine Eisensubstitution ist außerdem notwendig, wenn vor oder im Verlauf einer Therapie mit Erythropoetin ein funktioneller Eisenmangel auftritt. Dieser ist gekennzeichnet durch ausreichend vorhandenes Speichereisen in Kombination mit einer Erhöhung des Anteils hypochromer Erythrozyten im peripheren Blut als Ausdruck einer eisendefizitären Erythropoese. Die intravenöse Eisenapplikation ist dabei der oralen Gabe in Bezug auf Effektivität überlegen. Patienten mit normalen Eisenreserven oder Eisenüberschuss (Ferritin > $100 \mu \mathrm{g} / \mathrm{l}$ ) ohne Anhalt für eine eisendefizitäre Erythropoese sollten kein Eisen erhalten. Die erythropoetische Stimulation mit den derzeit verfügbaren pharmakologischen Produkten Epoetin alpha, Epoetin beta und Darbepoetin führt bei ACD in bis zu 100\% zu einem therapeutischen Erfolg, wenn gleichzeitig der jeweilige individuelle Eisenbedarf Berücksichtigung findet. Unter einer kombinierten Erythropoetin-Eisen-Therapie ist bei ACD in Assoziation mit Autoimmunerkrankungen wie z.B. der RA neben der Korrektur der Anämie eine Reduktion der Krankheitsaktivität

\section{Abstract \\ $\nabla$}

The anaemia of chronic disease is a concomitant phenomenon of various chronic intectious, neoplastic and autoimmune diseases. Whenever possible, the treatment of the underlying disease is the treatment of choice. When this is not feasible at present blood transfusion, iron therapy and the use of erythropoietic agents are further treatment options. Blood transfusions are reserved for the short-term use in the case of life-threatening anaemia $(\mathrm{Hb}<6.5 \mathrm{~g} / \mathrm{dL})$ and for the management of surgical interventions. Iron supplements are recommended when the ACD is accompanied by an iron deficiency with complete depletion of iron stores. Supplementation of iron is also required before or in the course of erythropoietin treatment when the ACD is associated with a functional iron deficiency. A functional iron deficiency is characterised by normal or even increased iron stores in combination with an increase of hypochromic red cells in the peripheral blood functioning as an indicator of an iron-deficient erythropoiesis. The intravenous administration of iron is more effective when compared to the oral route. Patients with ACD and normal or increased iron stores (serum ferritin $>100 \mu \mathrm{g} / \mathrm{L}$ ) without functional iron deficiency should not be treated with iron. The erythropoietic stimulation with the currently available agents epoetin alfa, epoetin beta and darbepoetin can result in a nearly $100 \%$ therapeutic response provided the individual iron requirements are considered. Using erythropoietin in combination with iron in patients with RA und ACD, it has been shown that besides the correction of the haemoglobin deficit also disease activity as well as health-related quality of life could be approved. The risk-benefit ratio of the treatment of ACD regarding the underlying disease has, however, not been fully evaluated so far. This is also true for the use of iron and erythropoietin in the treatment of ACD. 
und eine Verbesserung der Lebensqualität nachgewiesen worden. Insgesamt sind Risiken und Auswirkungen der Behandlung der chronischen Entzündungsanämie auf Verlauf und Prognose der jeweiligen Grundkrankheit jedoch noch nicht ausreichend untersucht. Ebenso bedürfen die Auswirkungen der Therapie mit Eisen und der Stimulatoren der Erythropese weiterer Evaluationen. Weitere therapeutische Optionen wie die Anwendung von Chelatbildnern oder anderen Substanzen mit regulierender Wirkung auf die zelluläre Eisenhomöostase sind aus präklinischen Forschungsergebnissen für den zukünftigen klinischen Einsatz bereits erkennbar. Für die Rheumaversorgung kann festgehalten werden, dass die ACD als Begleiterscheinung zahlreicher rheumatologischer Autoimmunerkrankungen heute auch ohne Sanierung der Grunderkrankung effektiv behandelt werden kann.

\section{Einleitung \\ $\nabla$}

Die chronische Entzündungsanämie (Anaemia of chronic disease - ACD) ist ein häufiges Begleitphänomen chronischer Krankheitsprozesse, wie Infektionen, chronische Nierenerkrankungen, Neoplasien und Autoimmunkrankheiten. Die ACD ist zumeist mild in ihrer Ausprägung und eng verknüpft mit Aktivität und Schwere der jeweiligen Grundkrankheit. Die Erythropoese ist hypoproliferativ, relativ refraktär gegenüber Erythropoetin und weist Anzeichen eines funktionellen Eisenmangels auf (s. Beitrag Prof. G. Weiss in dieser Ausgabe). Im Rahmen der Behandlung der jeweiligen Grundkrankheit kann sich z. B. durch arzneimittelbedingte Darmblutungen (NSAR) ein Eisenmangel mit der Entzündungsanämie kombinieren oder eine toxische Knochenmarkschädigung durch Chemotherapie oder Strahlentherapie die Anämie zusätzlich aggravieren (s. Beitrag Dr. U. Arndt in dieser Ausgabe).

Eine erfolgreiche Behandlung der Grundkrankheit beseitigt in der Regel auch die begleitende ACD und sollte stets als primäres therapeutisches Ziel gelten. Eine Heilung der Grundkrankheit ist jedoch oftmals bei Tumorerkrankungen, bei chronischer Niereninsuffizienz und bei Autoimmunerkrankungen, wie z.B. der rheumatoiden Arthritis nicht erreichbar. Die Indikation zu einer Therapie der ACD in diesen Fällen sollte nicht allein vom Ausmaß des Hämoglobindefizits abhängig gemacht werden. Patienten mit einem höheren Alter ( > 65 J.), Tumorpatienten unter Chemotherapie, Patienten mit Niereninsuffizienz unter Dialysebehandlung und auch Patienten mit rheumatoider Arthritis profitieren auch bei milder bis moderater Anämie (Hb zwischen 10 und $12 \mathrm{~g} / \mathrm{dl}$ ) mit einer Verbesserung ihrer Lebensqualität von einer Korrektur der Anämie [12, 28; s. Beitrag Dr. U. Arndt]. Trotz zunehmender Verbesserung des Verständnisses von Klinik und Pathogenese der ACD und trotz der Formulierung von Management Guidelines für ACD bei renaler Anämie und Tumoranämie wird in der klinischen Praxis der ACD insbesondere auch bei Erkrankungen des rheumatischen Formenkreises bis heute noch relativ wenig Aufmerksamkeit geschenkt $[2,8]$

Im nachfolgenden Übersichtsartikel sollen die verschiedenen therapeutischen Optionen vor allem am Beispiel der rheumatoiden Arthritis dargestellt und anhand der bisherigen klinischen Erfahrung evaluiert werden.
Further therapeutic options such as the use of iron chelating agents or other agents with an influence on the regulation of the cellular iron metabolism are in preclinical evaluation. It can, however, already be stated with regard to an appropriate management of patients with rheumatic diseases, regardless of the underlying disease, that ACD can be treated effectively.

\section{Therapie der Grundkrankheit}

$\nabla$

Proinflammatorische Zytokine wie z. B. TNF-alpha, Interleukin-1 (IL-1), Interleukin-6 (IL-6) und gamma-Interferon beeinflussen bei chronischen Entzündungsprozessen, wie sie z.B. mit der rheumatoiden Arthritis (RA), Morbus Crohn oder neoplastischen Prozessen assoziiert sind, direkt die Erythropoese indem sie die erythropoetische Stammzellproliferation hemmen, die Apoptose erythropoetischer Progenitorzellen fördern und inhibitorisch auf die Eisenfreisetzung aus Makrophagen wirken [2,10,17, $22,28]$.

In einer der ersten klinischen Studien zur Evaluation der therapeutischen Wirkung von Infliximab (cA2) haben Davis und Mitarbeiter [4] bereits1997 zeigen können, dass neben einer signifikanten Reduktion der Aktivität der Arthritis auch ein signifikanter, dosisabhängiger Hb-Anstieg erreicht werden kann. ( $\bullet$ Abb. 1). Die Autoren führten den beobachteten Hb-Anstieg auf eine Aufhebung der durch die proinflammatorischen Zytokine TNF-alpha und IL-6 bewirkte Proliferationshemmung der Erythropoese durch den TNF-alpha inhibierenden monoklonalen Antikörper Infliximab zurück. Papadaki et al. [18] haben die Aufhebung des inhibitorischen Effektes von TNF-alpha auf das erythropoetische Stammzellkompartment von Patienten mit chronischer Entzündungsanämie und RA durch Therapie mit dem monoklonalen Antikörper Infliximab mit in vivo/in vitro Stammzellexperimenten bestätigt. Ein ähnlicher Effekt auf die Hb-Konzentration bei RA mit chronischer Entzündungsanämie ist auch für Prednisolon beschrieben worden, das ebenfalls die Aktivität proinflammatorischer Zytokine signifikant inhibieren kann [13]. Dies gilt auch für die häufig angewandten DMARDs, wie Methotrexat, Leflunomid und Sulfasalazin. In einer 2008 veröffentlichten randomisierten, placebokontrollierten Studie zur therapeutischen Wirkung des humanisierten anti - Interleukin-6 Rezeptor Antikörpers Tocilizumab bei Patienten mit RA konnte ein signifikanter Anstieg der Hb-Konzentration in der Tocilizumab Gruppe im Vergleich zur Kontrollgruppe nachgewiesen werden [7]. Dieser Effekt des anti IL-6 Antikörpers auf die Entzündungsanämie kann vor allem auf eine Verbesserung der Eisenverfügbarkeit für die Erythropoese durch Hemmung der IL6 induzierten Hepcidin - Synthese zurückgeführt werden (s.a. Beitrag Prof. G. Weiss) Das klinisch inzwischen sehr erfolgreiche therapeutische Prinzip der Zytokin-Inhibition ist somit eine der in ihrer Wirkungsweise bereits partiell verstandene, klinisch effektive Therapie-Option zur Behandlung der chronischen Entzündungsanämie. Voraussetzung ist die diagnostische Abgren- 


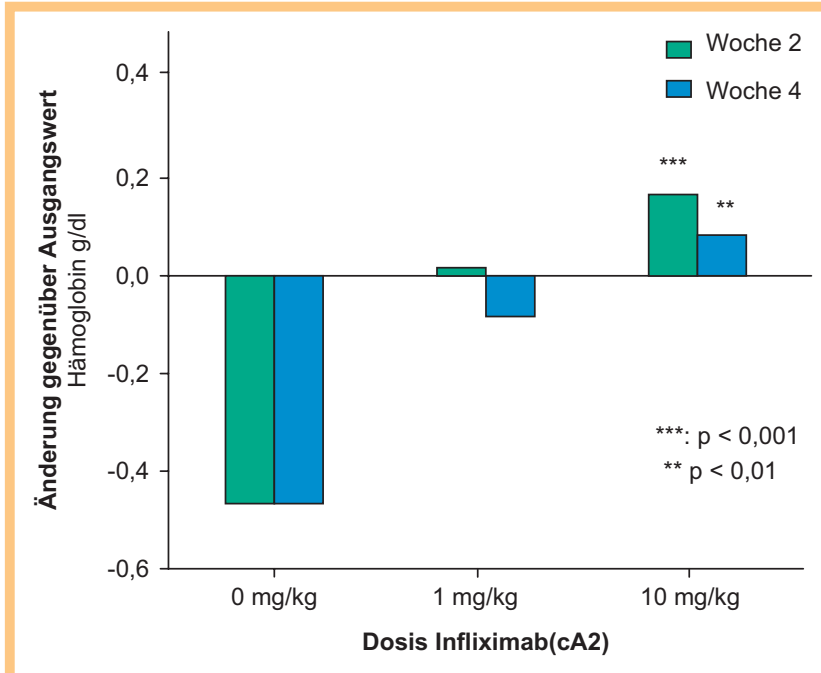

Abb. 1 Veränderungen der Hb-Konzentration 2 und 4 Wochen nach Therapie mit dem TNF alpha Antagonisten Infliximab (cA2) bei Patienten mit entzündlich aktiver rheumatoider Arthritis [4].

zung von anderen Anämie - Ursachen - vor allem von einem gleichzeitig vorhandenen Eisenmangel (s. Beiträge Prof. Schäfer; Dr. U. Arndt) und eine Indikation der Anwendung von ZytokinInhibitoren wie TNF-alpha Inhibitoren oder IL-1 bzw IL-6 Inhibitoren für die vorliegende Grundkrankheit.

\section{Bluttransfusion}

$\nabla$

Bluttransfusionen entweder als Vollblutkonserve oder in Form von Erythrozytenkonzentraten sind aufgrund der schnellen und effektiven therapeutischen Wirkung eine wichtige Option zur Therapie der ACD. Die Indikation zur Anwendung der Blutersatztherapie ist jedoch bei den unterschiedlichen Krankheitsentitäten, die mit der ACD assoziiert sein können, relativ unterschiedlich.

Im Vergleich zur renalen Anämie, zur Anämie bei fortgeschrittenem AIDS und zu Tumorerkrankungen ist die ACD bei rheumatischen Autoimmunkrankheiten selten schwer ausgeprägt. Die Hämoglobinkonzentration liegt bei RA Patienten zumeist bei 11,0-9,0 g/dl. Eine Indikation zu einer Substitutionstherapie mit Erythrozytenkonzentraten oder Vollblut besteht jedoch erst bei Hb-Konzentrationen unter 8,0 g/dl bzw. bei gleichzeitig bestehenden gravierenden Begleiterkrankungen wie z. B. einer koronaren Herzkrankheit bzw. vor geplanten chirurgischen Eingriffen.

Eine absolute Indikation besteht erst bei einer Hb-Konzentration $<6,5 \mathrm{~g} / \mathrm{dl}$ [28].

Bei der Indikationsstellung für eine Blutersatztherapie sind darüber hinaus stets auch die Transfusions-bezogenen Risiken, wie Volumenüberlastung, allergisch-anaphylaktische Risiken, Eisenüberladung und die Übertragung von Infektionen zu berücksichtigen [25]. Die nichtinfektiösen Risiken der Blutersatztherapie können überwiegend vermieden werden, wenn anstelle von Vollblut gewaschene Erythrozytenkonzentrate verwendet werden. Infektiöse Risiken, vor allem Virusübertragungen (HIV 1,2, HCV, HBV und HTLV I/II) stellen ein trotz Laborscreening noch immer vorhandenes Risiko dar, das auch bei der wichtigsten Indikation für eine Bluttransfusion, der präoperativen
Substitution, inzwischen große Vorbehalte sowohl bei den Patienten als auch den Chirurgen hervorruft. Die Alternative einer Eigenblutspende ist häufig bei entzündlich rheumatischen Erkrankungen wie der rheumatoiden Arthritis oder den Spondyloarthritiden gerade aufgrund der bestehenden Entzündungsanämie nicht möglich. Bluttransfusionen ohne gleichzeitige erythropoetische Stimulation mit Erythropoetin sind daher insgesamt betrachtet, vor allem bei den o.g. schweren, oft komplizierten Verlaufsformen der ACD in Verbindung mit Niereninsuffizienz, Neoplasien oder AIDS indiziert und kommen nur selten unter besonderen individuell bedingten Konstellationen im Verhältnis zu den ansonsten verfügbaren Therapiemodalitäten (s.u.) bei Erkrankungen aus dem rheumatischen Formenkreis in Betracht. Eine Langzeitbehandlung ausschließlich mit Blutersatz sollte auch bei den genannten klinischen Konstellationen wegen eines hohen Risikos für Eisenüberladung und Sensibilisierung gegenüber HLA-Antigenen nicht durchgeführt werden $[25,28]$.

\section{Eisentherapie}

\section{$\nabla$}

Eine orale oder parenterale Eisensubstitution ist bei chronischer Entzündungsanämie ohne gleichzeitig vorhandenen Eisenmangel bzw. ohne gleichzeitige Therapie mit Erythropoetin (s.u.) nicht indiziert. Es ist deshalb diagnostisch besonders wichtig, eine klare Abgrenzung gegenüber Eisenmangel vorzunehmen, (s. a. Beiträge Prof. Schäfer, S, Dr. U. Arndt.).

Oral appliziertes pharmazeutisches Eisen wird bei ACD nur in reduziertem Umfang absorbiert. Eisen ist darüber hinaus ein essentieller Wachstumsfaktor für Zellen jeder Art und vor allem auch für pathogene Mikroorganismen. Daher kann eine Eisensubstitution die einer Entzündungsanämie zugrunde liegende Infektion oder Krebserkrankung durch Wachstumsförderung der Tumorzellen oder der pathogenen Keime aktivieren bzw. Heilungsmechanismen behindern [9,27,28].Eine Eisensubstitution sollte deshalb nur bei gleichzeitig vorhandenem Eisenmangel vorgenommen werden ( $\bullet$ Tab. 2). Eine Eisentherapie ist nicht indiziert, wenn die Eisenreserven normal oder erhöht sind, d.h. die Serumferritin Konzentration $100 \mu$ g/oder mehr beträgt. In Verbindung mit einer Therapie der ACD mit rekombinantem humanen Erythropoetin dagegen ist eine Eisensubstitution sehr häufig indiziert und ggf. sogar eine essentielle Voraussetzung für einen optimalen therapeutischen Effekt, wie im folgenden Kapitel dargelegt wird.

\section{Erythropoetin}

Erythropoetin (EPO), ein 30,4 kD -Glykoprotein aus der Familie der hämatopoetischen Wachstumsfaktoren wird seit 1986 erfolgreich in der Therapie der renalen Anämie eingesetzt [5] Da bei der ACD schon seit längerem bekannt ist, dass eine im Verhältnis zum Ausmaß der Anämie zu geringe („blunted“) Erythropoetin-Sekretion und eine u.a. daraus resultierende Hypoplasie der Erythropoese zu den pathogenetischen Ursachen der Anämie gehören [14, 28;s. a.Beitrag Prof. G. Weiss] wurde bereits 2 Jahre nach Beginn der ersten klinischen Prüfung bei der renalen Anämie rekombinantes Erythropoetin auch bei zwei Patienten mit RA und chronischer Entzündungsanämie erstmals therapeutisch erprobt [16]. Im Gegensatz zur renalen Anämie blieb die Verwendung von EPO bei der chronischen Entzün- 
dungsanämie in Verbindung mit entzündlich rheumatischen Systemerkrankungen wie der RA zunächst umstritten. In nachfolgenden Anwendungen differierten die Ansprechraten bei relativ hohen EPO Dosen von 300 und bis zu $1250 \mathrm{IE} / \mathrm{kg}$ pro Woche sowohl s.c. wie i.v. mit $17 \%-90 \%$ in verschiedenen meist unkontrollierten Studien außerordentlich [14]. Bei anderen Erkrankunkungen wie z.B. Neoplasien unter Chemotherapie, entzündlichen Darmerkrankungen und HIV Infektionen, die ebenfalls mit einer ACD assoziiert sein können, wurde EPO ebenfalls mit überwiegend signifikant positivem Effekt auf die Anämie-Korrektur eingesetzt. Von der American Society of Clinical Oncology und der American Society of Hematology wurden Leitlinien für die Anwendung von EPO bei der Chemotherapie-assoziierten Anämie formuliert, in denen EPO Dosen von $150 \mathrm{IU} / \mathrm{kg}$ bis 300 $\mathrm{IU} / \mathrm{kg}$ dreimal wöchentlich s.c. bis zum Erreichen einer Hb-Konzentration von $>12,0 \mathrm{~g} / \mathrm{dl}$ empfohlen werden. Erythropetin in seinen derzeit verfügbaren Darreichungsformen Epoitin alfa, Epoetin beta und Darbepoetin wurde besonders bei der renalen Anämie und der Tumoranämie in großem Umfang eingesetzt. Das als letztes Derivat entwickelte Darbepoetin alpha hat bei vergleichbarer Wirksamkeit in der bei subcutaner Anwendung auf das Doppelte verlängerten Halbwertzeit den Vorteil, dass die Applikationsintervalle pro Dosis auf 1-2 Wochen verlängert werden können.

Die zu Beginn überwiegend negative Beurteilung des therapeutischen Effektes von EPO bei ACD in Assoziation mit entzündlich rheumatischen Systemerkrankungen wie z. B. der RA bezog sich auf eine Reihe von Fallstudien, in denen übersehen worden war, dass für einen optimalen therapeutischen Effekt von rekombinantem Erythropoetin (rHu-EPO) der individuelle Eisenstatus des Patienten berücksichtigt werden muss und ggf. eine zusätzliche Eisensubstitution erforderlich sein kann (s.u.).

Dieses Problem war bei der Therapie der renalen Anämie mit EPO bereits evident geworden und hatte $\mathrm{zu}$ entsprechenden Richtlinien geführt, die neben einer Steigerung des EPO Effektes auch zu einer Kosteneinsparung durch Dosisreduktion beitragen konnten [15].

Für die mit RA assoziierte Entzündungsanämie wurde vor allem in 2 Therapie-Studien [12,20] dieser Zusammenhang berücksichtigt und mithilfe einer zusätzlichen Eisensubstitution eine klinisch relevante Amämiekorrektur erzielt. Hinsichtlich einer optimalen EPO-Dosierung liegen bislang allerdings noch keine vergleichenden Studien für die Anwendung bei ACD vor.

In einer randomisierten, placebo-kontrollierten Studie haben Peeters et al. [20] 70 Patienten mit entzündlich aktiver RA und Entzündungsanämie rHu-EPO in einer initialen Dosierung von $240 \mathrm{IU} / \mathrm{kg}$ s. c. dreimal wöchentlich über einen Zeitraum von 52 Wochen verabreicht. In dieser Studie wurde Eisen fakultativ oral verabreicht, wenn die Serumferritin-Konzentration $<50 \mu \mathrm{g} / \mathrm{l} \mathrm{er-}$ reichte. Dies war bei $88 \%$ der Patienten der EPO-Gruppe zumeist im ersten Drittel der Studienperiode der Fall.

In der EPO - behandelten Patientengruppe erreichten 32 von 34 (94\%) eine normale Hb-Konzentration während dies nur bei 8 von 36 (22\%) Patienten in der Placebo-Gruppe der Fall war. Eine Normalisierung der Hb-Konzentration wurde im Durchschnitt nach 6 Wochen erreicht und konnte dann über insgesamt 52 Wochen mit reduzierter rHu-EPO Dosis (240 IU/kg s.c. 1 x wöchentlich) stabil erhalten werden. Darüber hinaus wurde mithilfe eines modifizierten Paulus Index [19], einem den ACR - Respons-Kriterien vergleichbarem Index, auch eine signifikante Reduktion der Krankheitsaktivität beobachtet. Am Ende der Studie hatten 38\% der Patienten in der EPO-Gruppe eine Paulus
$20 \%$ Response erreicht, während dies in der Placebogruppe nur bei $8 \%$ der Fall war.

Die Autoren stellten in der Diskussion ihrer Ergebnisse die Frage, ob aus der beobachteten Beseitigung der Anämie und der zusätzlich eingetretenen Reduktion der Krankheitsaktivität auch eine Verbesserung der Lebensqualität bei den Patienten erreicht wird und regten an, hierzu klinische Untersuchungen durchzuführen.

Eine solche Studie wurde von unserer Arbeitsgruppe [12] wenig später initiiert: Wir haben 30 Patienten mit entzündlich aktiver RA und Entzündungsanämie in einer unkontrollierten Studie mit $150 \mathrm{IU} / \mathrm{kg}$ rHu-EPO s.c. zweimal wöchentlich über 12 Wochen behandelt. Bei Vorliegen eines funktionellen Eisenmagels (Serumferritin $<50 \mu \mathrm{g} / \mathrm{l}$, Sättigung der TEBK $<15 \%$ und oder Anteil hypochromer Erythrozyten im peripheren Blut >10\% ) wurde zusätzlich Eisen in Form von $200 \mathrm{mg}$ Eisen-sucrose (Ferrum Vitis $\left.{ }^{\circledR}\right)$ einmal wöchentlich intravenös verabreicht. Nach 8,7 \pm 2,3 Wochen wurde bei allen Patienten mit diesem TherapieKonzept eine Normalisierung der Hb-Konzentration erzielt. In - Abb. 2 ist der Verlauf der mittleren Hb-Konzentration in der EPO-Therapiephase zusammen mit dem Anteil hypochromer Erythrozyten im peripheren Blut dargestellt. Innerhalb einer 3-

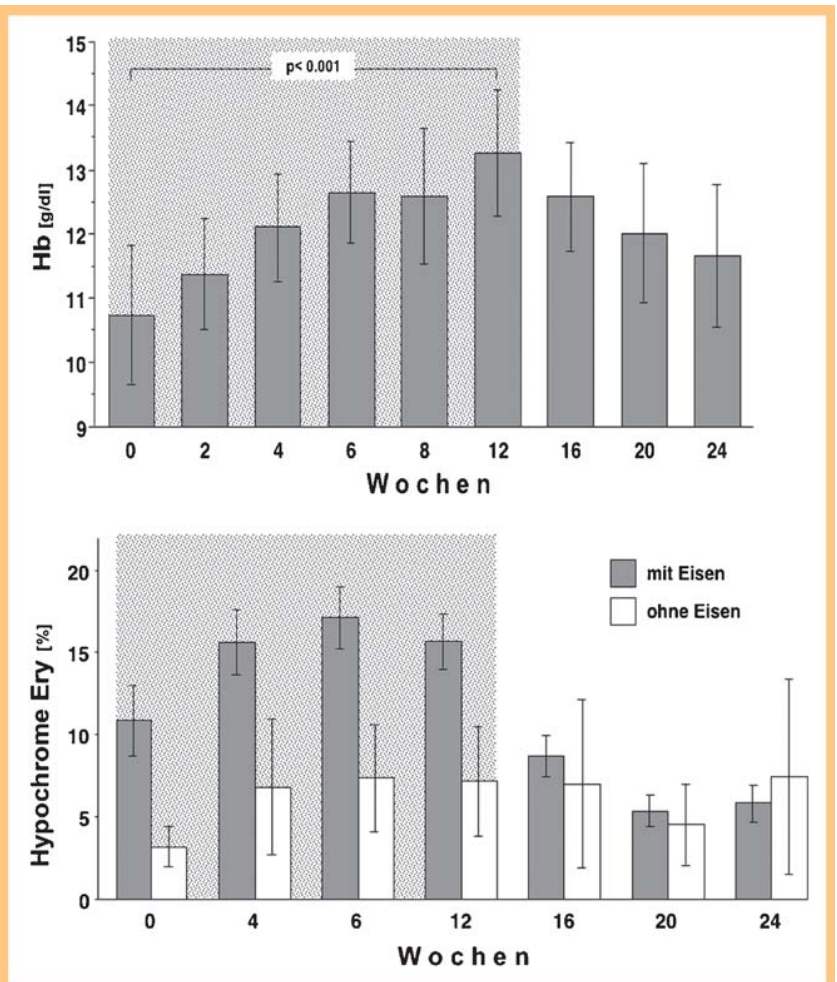

Abb. 2 Effekt von rekombinantem humanen Erythroetin auf die Hämoglobin-Konzentration ( $\mathrm{Hb}$ ) und den Anteil hypochromer Erythrozyten (hypochrome Ery) im peripheren Blut bei 28 Patienten mit entzündlich aktiver RA und ACD. Oberer Teil der Abbildung: Hämoglobinanstieg in 2 wöchentlichen Intervallen über 3 Monate unter Therapie mit $150 \mathrm{IU} / \mathrm{kg}$ rHu-EPO (Recormon ${ }^{\circledR}$ ) s. c. in Kombination mit 200 mg Eisen-Succrose (Ferrum Vitis) einmal wöchentlich i. v. bei Auftreten eines funktionellen Eisenmangels (s. a. Text).Schraffiertes Feld.: Therapiephase; Wochen 12-24: Nachbeobachtungsphase ohne rHuEPO/i.v. Eisentherapie. Unterer Teil der Abbildung: Anteil hypochromer Erythrozyten im Verlauf und nach rHu EPO/Eisentherapie: Graue Säulen: rHu EPO Therapie in Kombination mit i. v. Eisen ( $n=23)$; Weiße Säulen: rHu-Therapie ohne Eisensubstitution ( $n=5)$. Beachte: Unter rHuStimulation der Erythropoese nimmt der Anteil hypochromer Ery trotz Eisensubstitution weiter zu [12]. 
monatigen Nachbeobachtungsphase ohne rHu-EPO Therapie fällt die $\mathrm{Hb}$-Konzentration, wie 0 Abb. 2 zeigt, wieder nahezu auf den anämischen Ausgangswert zurück. Zusätzlich wurde in der Studie die Krankheitsaktivität mithilfe des Disease Activity Scors DAS 28 und dem RA Disease Activity Index (RADAI) bestimmt, eine monatliche Muskelkraftmessung vorgenommen und Müdigkeit (Multidimensional Assessment of Fatigue (MAF)) und Lebensqualität mittels der SF-36 Vitality Subscale und dem Health Assessment Questionnaire (HAQ) bestimmt (s.a. Beitrag Dr. U. Arndt). Es konnte sowohl eine signifikante Zunahme der Muskelkraft, wie auch eine Abnahme der Müdigkeit, eine Zunahme der Vitalität und eine Verbesserung der Funktionalität (HAQ) nachgewiesen werden. Die behandelten Patienten profitierten also messbar in Bezug auf eine Verbesserung ihrer Lebensqualität von der Beseitigung der ACD.

Recombinantes humanes Erythropoetin weist als humanisiertes Protein wenig Nebenwirkungen auf. Vermieden werden muss wegen möglicher Herzkreislaufkomplikationen eine Überkorrektur der Anämie ( $\mathrm{Hb}>13,0 \mathrm{~g} / \mathrm{dl}$ bei Frauen; >14,0 g/dl bei Männern). Es wird eine Ziel-Hb-Konzentration von 12,0 g/dl empfohlen [28].

\section{Erythropoetin und Eisenstatus \\ $\nabla$}

Eisen muss als essentielles Substrat der Haemoglobinsynthese bei der Stimulation der Erythropoese mittels rHu-EPO in ausreichender Menge und in bioverfügbarer Form zur Verfügung stehen. Dies ist sowohl bei der renalen Anämie wie bei Entzündungsanämie in Verbindung mit Erkrankungen wie RA, entzündlichen Darmerkrankungen oder Neoplasien infolge von Einschränkungen in der oralen Eisenaufnahme und aufgrund einer limitierten Eisenfreisetzung aus dem Eisenspeicherkompartment nicht im erforderlichen Umfang der Fall [28]. Es resultiert daraus häufig trotz ausreichend gefüllter Eisenspeicher eine eisendefizitäre Erythropoese. Diese Konstellation wird in Abgrenzung zum klassischen Eisenmangel als „funktioneller Eisenmangel“ bezeichnet, ist charakteristisch für die hypoproliferative Entzündungsanämie und kann ebenfalls auch durch den Stimulationseffekt des rHu-EPO induziert werden [11,14]. Diese im Zusammenhang mit der rHu-EPO - Therapie der renalen Anämie ermittelten Erkenntnisse haben dazu geführt, dass eine begleitende intravenöse Eisentherapie heute Standard in der Therapie der renalen Anämie mit rHu-EPO geworden ist [1].

Der Befund des funktionellen Eisenmangels ist charakterisiert durch eine Serumferritin Konzentration $>100 \mu \mathrm{g} / \mathrm{l}$, eine Sättigung der TEBK $<20 \%$, einen Anstieg der Fraktion hypochromer Erythrozyten auf $>10 \%$ im peripheren Blut. Zusätzliche Informationen über die „interne“ Eisenversorgung der Erythropoese liefern die Bestimmungen des löslichen Transferrinrezeptors
(sTrfR), des Zink Protoporphyringehalts (ZnPP) der Erythrozyten und die Messung der Hämoglobinkonzentration der Retikulozyten $(\mathrm{cHr})$ [11; s. a. Beitrag Profs. Schaefer].

In einer Analyse der genannten Laborparameter bezüglich ihrer Eignung zur Detektion eines funktionellen Eisenmangels im Verlauf einer rHu-EPO Therapie bei RA mit Entzündungsanämie konnten wir zeigen, dass mit einem Index, gebildet aus sTrfR/Fn die beste Vorhersage ( $100 \%$ Spezifität, $86,7 \%$ Sensitivität) für einen funktionellen Eisenmangel erreicht wird. Im Verlauf einer kombinierten Therapie mit rHu-EPO und i.v. applizierter EisenSuccrose (s.o.) wurde bei 28 (83\%) von 30 Patienten im Verlauf der Therapie eine eisendefizitäre Erythropoese dokumentiert [3]. Intravenös verabreichtes Eisen in Verbindung mit rHu-EPO ist effektiver als oral verabreichtes Eisen. In einer Studie von Fishbane et al. [6] führte eine intravenöse Applikation von $2 \mathrm{x}$ $100 \mathrm{mg}$ Eisen zweimal wöchentlich im Vergleich zu oraler Eisentherapie bei renaler Anämie zu einer Einsparung von 46\% der rHu-EPO Dosis, die erforderlich war um einen Hämatokrit zwischen $30 \%$ und 34\% aufrechtzuerhalten. Eine Berücksichtigung des Eisenbedarfs bei rHu-EPO Therapie kann also auch, wie schon erwähnt, den rHu-EPO Bedarf senken und zur Kostenreduktion beitragen. Für die ACD bei entzündlich rheumatischen Erkrankungen wie der RA fehlen solche Vergleichstudien bisher allerdings noch. Intravenös verabreichtes Eisen kann zu anaphylaktischen Reaktionen führen. Bei Eisen-Dextran Präparationen ist dies bei $0,5-1 \%$ der behandelten Patienten der Fall. Deutlich seltener treten diese Nebenwirkungen bei Eisen (III) Hydroxyd - Sucrose (z. B. Ferrum Vitis ${ }^{\circledR}$, Venofer ${ }^{\circledR}$ ) auf, weshalb diese Präparationen bevorzugt eingesetzt werden [12].

\section{Erythropoetin und Eigenblutspende bei entzündlich rheumatischen Erkrankungen}

Patienten, die sich einem chirurgischen Eingriff unterziehen müssen, weisen in 30-50\% eine präoperative Anämie auf. In der postorperativen Phase ist der Anteil anämischer Patienten noch deutlich höher. Da eine perioperative Anämie bei chirurgischen Patienten die Prognose durch eine erhöhte Infektrate und erhöhte Mortalität negativ beeinflusst, werden vielfach unterschiedliche perioperative Strategien zur Vermeidung oder Korrektur von Anämien angewandt.

Die Möglichkeit der Übertragung von Infektionskrankheiten durch Bluttransfusionen und die Möglichkeit immunmediierter Komplikationen (s.o.) hat vor allem mit Blick auf Hepatitis und HIV Infektionen in den achtziger Jahren des letzten Jahrhunderts zu einem deutlichen Anstieg der Indikation bzw. der Anforderung für Eigenblutspenden insbesondere bei elektiven chirurgischen Eingriffen geführt. Alternativ zur Eigenblutspende wird die Retransfusion des operativen Blutverlustes und das Verfah-

Tab. 1 Laborindikatoren zur Differenzierung von Eisenmangelanämie und funktionellem Eisenmangel.

\begin{tabular}{|c|c|c|c|c|}
\hline Parameter & Normwerte & Entzündungs-Anämie & Eisenmangel Anämie & Funktioneller Eisenmangel \\
\hline Knochenmark Eisen & ++-+++ & ++-++++ & 0 & ++-++++ \\
\hline $\operatorname{TEBK}(\mu \mathrm{g} / \mathrm{dl})$ & 33030 & erniedrigt-normal & erhöht & erniedrigt-normal \\
\hline Sättigung der TEBK (\%) & $35 \pm 15$ & erniedrigt & erniedrigt & erniedrigt \\
\hline Serumferritin $(\mu \mathrm{g} / \mathrm{l})$ & $22-310$ & normal-erhöht & erniedrigt & normal-erhöht \\
\hline Serumtransferrinrezeptor* (mg/l) & $2,9-7,1$ & normal & erhöht & erhöht \\
\hline Hypochrome Ery (\%) & $<10$ & normal & erhöht & erhöht \\
\hline Transferrinrezeptor-Ferritin-Ratio & $<0,1$ & normal & erhöht & erhöht \\
\hline
\end{tabular}

*unterschiedlich definierte Normbereiche [24] 
Tab. 2 Therapeutische Optionen für die Behandlung der chronischen Entzündungsanämie (nach Weiss und Goodnough [28]).

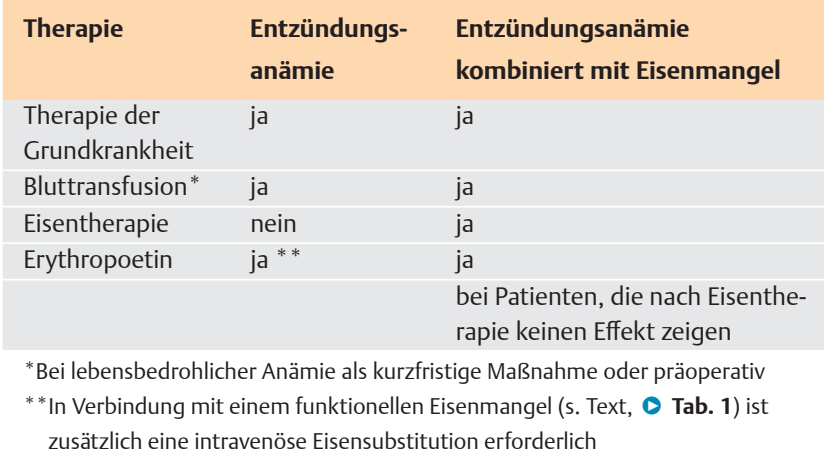

ren der isovolämischen Hämodilution - besonders bei geringem zu erwartenden Blutverlust, in der chirurgischen Praxis angewandt $[21,26]$. Für Patienten mit entzündlich rheumatischen Erkrankungen wie z.B. RA, Psoriasis Arthritis oder Spondyloarthritiden ist aufgrund des gleichzeitigen Vorhandenseins einer chronischen Entzündungsanämie eine Eigenblutspende präoperativ aber oft nicht durchführbar. Auch für diese spezifische Indikation ist rHu-EPO in Kombination mit einer Eisensubstitution geeignet, durch Steigerung der Erythropese eine Eigenbluspende zu ermöglichen oder alternativ die vorhandene Anämie präoperativ durch alleinige Stimulation mit rHu Epo und i.v. Eisen zu korrigieren $[8,14]$. Erythropoetin kann auch zur rascheren postoperativen Rekonvaleszenz durch Korrektur der durch den Eingriff induzierten Anämie beitragen. Es ist bisher nicht gezeigt worden dass Bluttransfusionen die Morbidität oder Mortalität operierter Patienten verbessert, während dies für die alternative perioperative Therapie mit Erythropoetin z.B. bei herzchirurgischen Eingriffen nachgewiesen worden ist [30] ( $\bullet$ Tab. 1).

\section{Schlussfolgerungen und Ausblick \\ $\nabla$}

Basierend auf den in jüngerer Zeit erweiterten Erkenntnissen über die Pathophysiologie der chronischen Entzündungsanämie und der Regulation der Eisenhomöostase stehen heute wirksame Therapiestrategien zur Verfügung, die es erlauben diese Anämie erfolgreich zu behandeln ( $\bullet$ Tab. 2).

Autoimmunologische Systemerkrankungen des rheumatischen Formenkreises können heute mit Zytokin-Inhibitoren, B-Zell depletierenden Antikörpern und den CTLA-4 Antagonisten signifikant besser behandelt werden. Darüber hinaus kann bei diesen Erkrankungen durch Stimulation der Erythropoese mit Erythropoetin die Entzündungsanämie beseitigt werden. Eine wichtige Voraussetzung für eine erfolgreiche Therapie mit Erythropoetin ist eine adäquate Versorgung der Erythropoese mit bioverfügbarem Eisen. Die Beseitigung der Anämie verbessert die Lebensqualität der Patienten und reduziert die Krankheitsaktivität.

Es bleibt bislang noch ungeklärt, ob z. B. Erythropoetin und phamazeutisches Eisen den Verlauf von Tumorerkrankungen und Infektionen auch negativ beeinflussen können [28]. Es steht, wie im Beitrag von Prof. G. Weiss in dieser Ausgabe ausgeführt wird, auch zur Diskussion, ob bei der ACD in Verbindung mit Autoimmunerkrankungen der mit der Anämie verbundene Effekt der Restriktion der Eisenfreisetzung aus Makrophagen nicht auch als natürlicher Beitrag zur Verbesserung der Infektresistenz und Immunkompetenz anzusehen ist. Weitere prospektive kontrol- lierte klinische Untersuchungen über den Effekt der angewandten Therapien zum Management der ACD erscheinen daher notwendig.

Mögliche neue Strategien zur Regulierung dieser Probleme bei der Entzündungsanämie in Verbindung mit unterschiedlichen Grundkrankheiten zeichnen sich bereits ab. So könnte z.B. die Anwendung von Chelatbildnern [23,29], von Hepcidin-Antagonisten oder neuen Zytokin-Inhibitorern [7,28] zu einer effektiven Stimulation der Erythrpoese ohne negative Auswirkungen auf die Infektabwehr und Immunkompetenz führen.

Interessenkonflikt: Nein

\section{Literatur}

1 Adamson JW, Eschbach JW. Erythropoietin for end-stage renal disease. N Engl J Med 1998; 339: 625-627

2 Alvarez-Hernandez X, Liceaga J, McKay IC et al. Induction of hypoferremia and modulation of macrophage iron metabolism by tumor necrosis factor. Lab. Invest 1989; 61: 319-322

3 Arndt U, Kaltwasser JP, Gottschalk R et al. Correction of iron - deficient erythropoiesis in the treatment of anaemia of chronic disease with recombinant human erythropoietin. Ann Hematol 2005; 84: 159166

4 Davis D, Charles PJ, Potter A et al. Anaemia of chronic disease in rheumatoid arthritis: in vivo effects of tumor necrosis factor $\alpha$ blockade. Brit J Rheumatol. 1997; 36: 950-956

5 Eschbach JW, Egrie JC, Downing MR et al. Correction of the anaemia of end-stage renal disease with recombinant human erythropoietin. Results of a combined phase I and II clinical trial. N Eng J Med 1987; 316: 73-78

6 Fishbane S, Frei GL, Maesaka J. Reduction in recombinant human erythropoietin doses by the use of chronic intravenous iron supplementation. Am J Kidney Dis 1995; 26: 41-46

7 Genovese MC, McKay JD, Nasonov EL et al. Interleukin-6 receptor inhibition with Tocilizumab reduces disease activity in rheumatoid arthritis with inadequate response to diasese modifying antirheumatic drugs. Arthrits and Rheumatism 2008; 58: 2968-2980

8 Goodnough $L T$, Monk TG. Erythropoietin in perioperative setting. Clin Orthop Relat Res. 1998; 357: 82-88

9 Gordeuk VR. Iron therapy and the anaemia of chronic disease. In: Weiss G, Gordeuk VR, Hershko Ch, Eds. Anaemia of chronic disease. Boca Raton, Taylor \& Francis; 2005; 381-396

10 Jongen-Lavrencic $M$, Peeters HRM, Wognum A et al. Elevated levels of inflammatory cytokine. J. Rheumatol 1997; 24: 1504-1509 -(v)

11 Kaltwasser JP, Gottschalk R. Erythropoietin and iron. Kidney International 1999; 55 (suppl.69): 49-56

12 Kaltwasser JP, Kessler U, Gottschalk R et al. Effekt of recombinant human erythropoietin and intravenaous iron on anaemia and disease activity in rheumatoid arthritis. J Rheumatol 2001; 28: 2430-2436

13 Kendall $R$, Wasti A, Harvey A et al. The relationship of haemoglobin to serum erythropoietin concentrations in the anaemia of rheumatoid arthritis: the effect of oral prednisolone. Br. J. Rheumatol 1993; 32: 204-208

14 Kessler U, Kaltwasser JP. Humanes rekombinantes Erytropoietin in der Therapie der Anämie bei rheumatoider Arthritis, Akt. Rheumatol 2001; 26: 15-21

15 Locatelli F, Aljma P, Barany P et al. Revised European best practice guidelines for the management of anaemia in patients with chronic renal failure. Nephrol Dial Transplant 2004; 19 (Suppl 2): ii2-ii5

16 Means RT, Olsen NJ, Krantz SB et al. Treatment of the anaemia of rheumatoid arthritis with recombinant human erythropoietin: Clinical and in vitro studies. Arthritis Rheum 1989; 32: 638-642

17 Means RT Jr, Krantz SB. Inhibition of human erythroid colony-forming units by $\gamma$-interferon can be corrected by recombinant human erythropoietin. Blood 1991; 78: 2564-2567

18 Papadaki HA, Kritikos HD, Valatas $V$ et al. Anaemia of chronic disease in rheumatoid arthritis is associated with increased apoptosis of bone marrow erythroid cells: improvement following anti-tumor necrosis factor-alpha antibody therapy. Blood 2002; 100: 474-482

19 Paulus HB, Egger MJ, Ward JR et al. Cooperative systemic studies of rheumatic diseases group. Analysis of improvement in individual rheumatoid arthritis patients treated with disease-modifying antirheumatic drugs, based on the findings in patients treated with placebo. Arthtis Rheum 1990; 33: 477-484 
20 Peeters HRM, Jongen-Lavrencic M, Vreugdenhil G et al. Effect of recombinant human erythropoietin on anaemia and disease activity in patients with rheumatoid arthritis and anaemia of chronic disease: a randomised placebo controlled double blind 52 weeks clinical trial. Ann Rheum Dis 1996; 55: 739-744

21 Penner M, Sibrowski W, Fingerhut D et al. Eigenblutspende und isovolämische Hämodilution - Indikation und praktsche Durchführung. Infusionsther Tramsfusionsmed 1993; 20: 307-315

22 Roodman GD. Mechanism of erythroid suppression in the anaemia of chronic disease. Blood Cells 1987; 13: 171-184

23 Sohn YS, Breuer W, Munnich A et al. Redistrbution of accumulated cell iron: a modality of chelation with therapeutic implications. Blood 2008; 111: 1690-1699

24 Thomas L. Löslicher Transferrinrezeptor (sTfR). In: Thomas L, Hrsg. TH Bools Verlagsgesellschaft mbH Frankfurt am Main. Labor und Diagnose; 1998; 289-291
25 Vamvakas EC. Blood Transfusion. In: Weiss G, Gordeuk VR, Hershko $\mathrm{Ch}$, (eds). Anaemia of chronic disease. Boca Raton, Taylor \& Francis; 2005; 397-435

26 Walpoth BH, Schwaller J, Hofstetter $H$ et al. Current problems with autologous blood supply. Infusionsther Transfusionsmed 1993; 20: 316-326

27 Weinberg ED. Iron withholding: a defense against infection and neoplasia. hysiol Rev 1984; 64: 65-102

28 Weiss G, Goodnough LT. Anemia of chronic disease. N Engl J Med 2005; 352: 1011-1023

29 Weiss G. Iron caught on the shuttle. Blood 2008; 111: 980

30 Yazicioglu L, Eryilmaz S, Sirlak $M$ et al. Recombinant human erythropoietin in cardiac surgery. Thorac Cardiovasc Surg 2001; 122 (4): 741-745 